\title{
STEM-in-SEM and Cryo-EM Comparison using Simulation and Experiments for Interleukin 17A-FAB Complexes on Graphene
}

\author{
Jennifer Carpena-Núñez ${ }^{1}$, Cheri Hampton ${ }^{2}$, Stewart Koppell ${ }^{3}$, Mark Kasevich ${ }^{3}$ and Lawrence Drummy ${ }^{4}$ \\ ${ }^{1}$ UES Inc., United States, ${ }^{2}$ UES, Inc., Brookville, Ohio, United States, ${ }^{3}$ Physics Department, Stanford University, \\ United States, ${ }^{4}$ Air Force Research Laboratory, Materials and Manufacturing Directorate, United States
}

The development and subsequent growth of super resolution optical microscopy and cryo-electron microscopy have brought on a new era for microscopists and biochemists.[1,2] These techniques, along with conventional electron imaging techniques, e.g., TEM, low voltage EM, and STEM-in-SEM, have enabled the visualization of highly beam-sensitive biomolecules with unprecedented resolution. They, however, do not come without challenges; labor-intensive sample preparation, biochemical optimization, and additional imaging optimization (to name a few) can discourage the 'faint of heart'. In this study, we combine a series of electron microscopy and simulation techniques to understand the ideal imaging regime and 'path-of-least-resistance' for imaging biomolecules. In this work, we image and simulate IL-17A-Fab complexes, a small protein Interlukin-17A and its corresponding IgG1 antibody Fabs. [3, 4] These biomolecules are inflammatory markers of relevance for human-health monitoring.

We examined the IL-17A-Fab complexes on graphene surfaces using cryo-electron microscopy, conventional electron microscopy (TEM), low-voltage electron microscopy (e.g., STEM-in-SEM), multi-pass electron image simulations, and STEM image simulations. Graphene provided an ultrathin support and graphene-biomolecule interactions have technological relevance for biosensor applications in ongoing studies at AFRL.[5] Cryo-electron microscopy was used to benchmark the image resolution.[6] We proceeded to comparing these to conventional TEM images (e.g., stained and unstained samples imaged at 200kV; Fischer Scientific Talos), with 'low voltage' TEM images (imaged at 80kV; Fischer Scientific Talos), and with STEM-in-SEM images (imaged at 5-30kV and detector conditions yielding SEM, bright field STEM, and dark field STEM; Zeiss Gemini). In particular, low voltage EM and STEM-in-SEM are focal for our work. Their attractiveness lies in their ease of use, their continuously-improving image resolution, their continuously-expanding imaging modes, and their ability to yield STEM images below the beam-damage threshold all-the-while reducing electron charging and increasing image contrast.[7-10] Although STEM-in-SEM images were not able to match the image quality obtainable through cryoEM, we found that it provided fast-throughput screening and image quality comparable to that achievable using conventional TEM in a fraction of the time.

Meanwhile, image simulations based on the known structures of these proteins were used to facilitate experimental screening, i.e., to forecast expected image quality and image projections.[11] STEM simulations were performed using Prismatic -an open-source image simulation software.[12] These simulations were executed using imaging conditions analogous to experimental conditions: accelerating voltage in the 5-200kV range, varying defocus, and probe alpha limit and spherical aberration values corresponding to the instrument parameters (e.g., Fischer Scientific Talos TEM and Zeiss Gemini). We also simulated images using various virtual detector angles to yield bright field, annular bright field, and dark field STEM images. Lastly, we simulated IL-17A and Fabs supported on graphene, using various orientations to simulate the possible specimen/support configurations and guide experimental imaging. Figure 1 depicts a defocus simulation series of IL-17A.

Lastly, we performed simulations of multi-pass electron microscopy developed at Stanford University.[13, 14] These simulations were also used to understand the attainable resolution for IL-17A from multi-pass electron microscopy. This novel technique (currently in development) is foreseen to alleviate beam-damage and propel bioimaging in the next few years.[15] Future efforts for this study will therefore focus on the implementation of multipass microscopy, and on the continued work for TEM image optimization, STEM-in-SEM image optimization, and phase contrast simulations. 


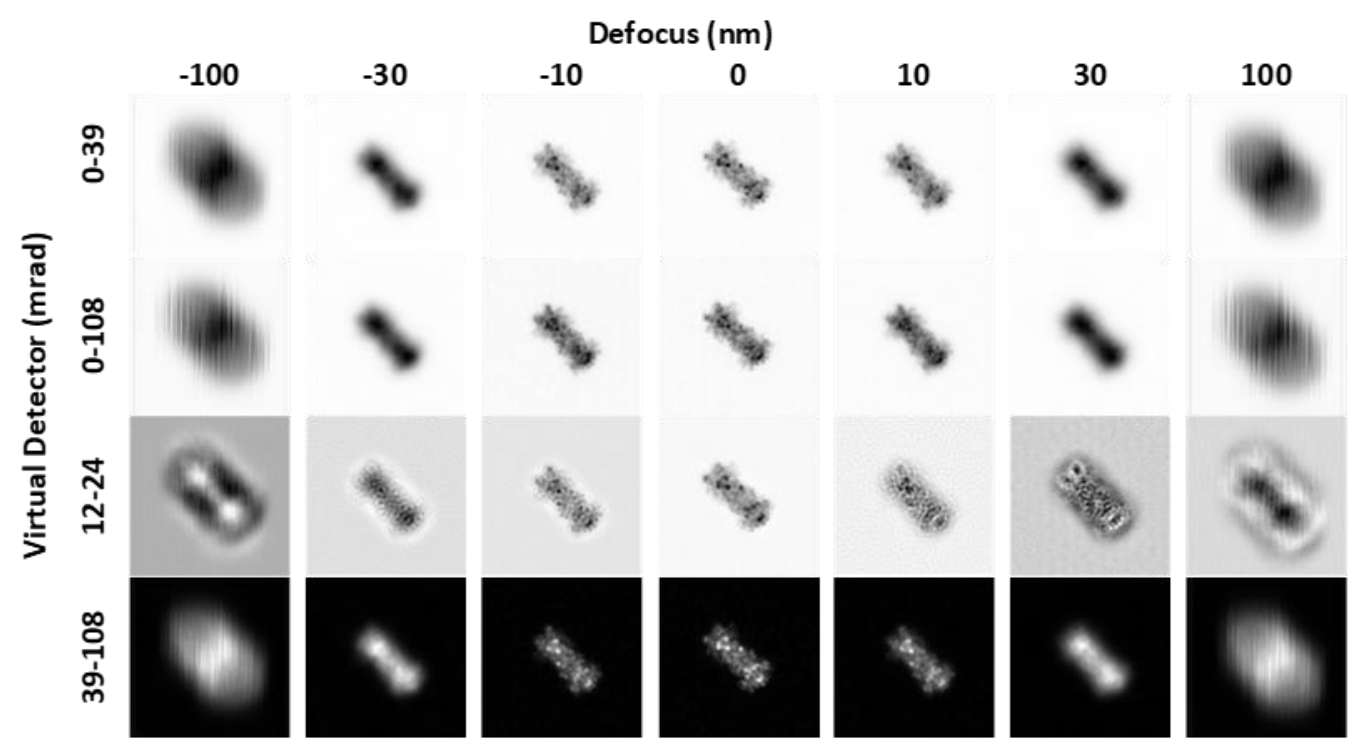

Figure 1. STEM image simulations of IL-17A on graphene. The array depicts a defocus series using an acceleration voltage of $5 \mathrm{kV}$ and probe alpha limit of $32 \mathrm{mrad}$.

\section{References}

[1] https://www.nobelprize.org/prizes/chemistry/2014/press-release/

[2] https://www.nobelprize.org/prizes/chemistry/2017/press-release/

[3] C Zenobia and G Hajishengallis, Periodontol 200069 (2015), p. 142.

[4] S Wu et al., Structure 20 (2012), p. 582.

[5] S Kim et al., ACS Appl Mater Interfaces 11 (2019), p. 13927.

[6] CM Hampton et al., Nat Protoc 12 (2017), p. 150.

[7] CG Golding et al., Sci Rep 6 (2016), p. 26516.

[8] L Zarraoa, MU Gonzalez and AS Paulo, Sci Rep 9 (2019), p. 16263.

[9] J Bacovsky, MethodsX 5 (2018), p. 1033.

[10] GW Gang et al., Sci Rep 10 (2020), p. 4868.

[11] https://www.rcsb.org/

[12] https://prism-em.com/

[13] https://web.stanford.edu/group/kasevich/cgi-bin/wordpress/?page_id=615

[14] T Juffmann et al., Sci Rep 7 (2017), p. 1699.

[15] SA Koppell et al., Ultramicroscopy 207 (2019), p. 112834. 